\title{
El Rendimiento Empresarial De Las Asociaciones En Un Marco De La Economía Popular Y Solidaria: Estudio En Un Programa Gubernamental
}

\section{Business Performance Of The Member Associations In A Framework Of The Popular And Solidarity Economy: Study In Government Program}

Hermel David Ortiz¹, Elsy Marcela Alvarez²

\begin{abstract}
1. Economista, Magister en Gerencia Empresarial. Docente en la Facultad de Contabilidad y Auditoría de la Universidad Técnica de Ambato, hd.ortiz@uta.edu.ec

2. Economista, Magister en Gestión Financiera. Docente en la Facultad de Contabilidad y Auditoría de la Universidad Técnica de Ambato,em.alvarez@uta.edu.ec
\end{abstract}

\section{Resumen}

Esta investigación analiza la situación de las actividades empresariales bajo un programa gubernamental buscando el bienestar de las asociaciones, satisfaciendo necesidades y generando fuentes de empleo, originándose una base para el rendimiento de las organizaciones; la economía popular y solidaria, que permite trabajar de forma ordenada obteniendo beneficios tanto para la entidad como para las personas naturales y al mismo tiempo favoreciendo al desarrollo del país. A través de este análisis se podrá determinar si es posible obtener un rendimiento. Este estudio se realizó a 117 integrantes del Programa Hilando el Desarrollo, programa gubernamental impulsado por el Gobierno del Ecuador.

Palabras claves: economía social y solidaria, asociatividad, rendimiento empresarial.

Abstract

This research analyzes the situation of business activities under a government program seeking the welfare of the associations, satisfying needs and creating sources of employment, creating a basis for the performance of the organizations; the popular and solidary economy, It allows to work in an orderly manner, obtaining benefits both for the institution and for natural persons and at the same time the development of the country. Through this analysis you can determine if it is possible to obtain a business returns. This study was conducted about 117 members of government program launched by the Government of Ecuador.

Keyworks: social economy and solidarity, associativity, business returns. 


\section{Introducción}

La búsqueda por obtener rendimientos empresariales ha motivado a los talleres artesanales tener como opción la asociatividad. La asociatividad para Castellanos (2010) crea condiciones de igualdad para todos los participantes, teniendo como resultado principal el cumplimiento de sus objetivos y un incremento de la rentabilidad y una alta cuota de mercado.

Unos de los temas más críticos es la Asociatividad, Castellanos (2010) menciona que la Asociatividad permite estimular las potencialidades y capacidades individuales, para así lograr un mejor posicionamiento, en los mercados y afrontando la incertidumbre que plantea el entorno. Los potenciales que poseen las asociaciones será una de las determinantes para su competitividad. El estudio de los factores y su distinta combinación potenciales determinan que unas empresas sean más competitivas que otras (Rumelt, 1991). La consideración de que una empresa posee una competitividad y ocupa una posición de mercado única y superior en términos de coste o diferenciación de sus productos (Porter, 1991). En busca de mejorar los rendimientos los talleres artesanales han explorado en el campo de la asociatividad. La rentabilidad por parte de Holland (1975) menciona que: considerando su terminología, rentabilidad se obtiene a través del planteamiento de dos tipos de aprendizaje la exploración y la explotación. Refiriéndose a la exploración como la búsqueda, la variación, el riesgo, la experimentación, la flexibilidad, el descubrimiento y la innovación. En cambio Real (2006) argumenta que la explotación se relaciona con la eficiencia, la selección y la ejecución. Siendo el pilar fundamental de las empresas para la ejecución de sus actividades cotidianas.

\section{Desarrollo}

\subsection{Asociatividad}

La Asociatividad se define como un mecanismo de cooperación entre empresas pequeñas y medianas, en donde cada empresa participante, manteniendo su independencia jurídica y autonomía gerencial, decide voluntariamente participar en un esfuerzo conjunto con los otros participantes para la búsqueda de un objetivo común. Rosales (1997) menciona que: por consecuencia la Asociatividad se convierte en una opción válida y necesaria para las pequeñas y medianas empresas unidas de manera permanente y voluntaria que puedan compartir sus esfuerzos y conocimientos. Para poder resistir no solo en un nuevo escenario económico, sino también, a cambios estructurales más eficientes (Maldovan \& Dzembrowski, 2009). 
La Asociatividad crea condiciones de igualdad para todos sus participantes, de la misma manera permite la interrelación con su entorno empresarial. Las empresas asociativas tienen el potencial de favorecer el desarrollo de los espacios rurales en cuanto pueden consolidar economías de escala, reducir costos de transacción, acceder a servicios técnicos y apoyos a la gestión. (Bedregal, 2014). Las ventajas de la Asociatividad son evidentes. Sin embargo, el paso más complicado y decisivo en la construcción de la misma se muestra en el momento no solo de compartir costos, sino también información y conocimiento.

En tal sentido la necesidad de impulsar un proceso de Asociatividad empresarial es importante, no solamente por todas las ventajas mencionadas que son evidentes, sino también como una estrategia colectiva que permita promover un cambio en la matriz productiva del país, con el propósito de romper el paradigma empresarial capitalista (individualista) y construir un sistema dinámico en el sector empresarial que cree las condiciones necesarias que conlleven hacia el desarrollo endógeno definido como la capacidad de liderar y conducir su propio desarrollo (Bedoya, 2005) del sistema productivo actual.

La Asociatividad puede adoptar diversas modalidades, dependiendo el objetivo por el cual se produce, cada una de ellas implican diversas formas de participación generando mayor o menor responsabilidad. Mencionando Vegas (2008) que los mismos que pueden ser:

- Redes de Cooperación.- Conjunto de empresas que comparten información

- Articulación Comercial.- Relación entre empresas ofertantes y demandantes.

- Alianza de cadenas Productivas.- Cada agente realiza una etapa distinta del proceso.

- Alianza en Clúster.- Comprende no solo empresas sino también instituciones.

- Join Venture.- Constitución de una nueva sociedad.

- Consorcios.- acuerdo entre varias empresas para es desarrollar una actividad económica.

Las diferentes modalidades de asociarse planteadas tienen un único fin que es la mejora del nivel competitivo de las pymes. Ya que al ser una empresa competitiva está comprometida a mejorar constantemente su servicio o producto para la satisfacción del cliente. A nivel de América Latina las pymes son consideradas como una fuente de ingreso y empleo, por tanto se fomenta la integración empresarial con el fin de alcanzar empresas productivas. En el cuadro expuesto a continuación muestra los beneficios de las pymes al decidir voluntariamente ser parte de una asociación. 


\begin{tabular}{|c|c|}
\hline Aspectos & Beneficios \\
\hline Aspecto Financiero & $\begin{array}{l}\text { - Acceso al financiamiento. } \\
\text { - Compras conjuntas de materia prima. } \\
\text { - Reducción de costos. } \\
\text { - Acceso a tecnología que requiere altos costos. } \\
\text { - Importación de bienes y servicios. }\end{array}$ \\
\hline $\begin{array}{l}\text { Razones } \\
\text { Organizacionales }\end{array}$ & $\begin{array}{l}\text { - Aumento del poder de negociación con los proveedores, gobierno local y clientes. } \\
\text { - Mejorar en los procesos productivos. } \\
\text { - Incorporación de capital humano. } \\
\text { - Establecimiento de alianzas estratégicas. } \\
\text { - Aprendizaje colectivo. } \\
\text { - Mejora la capacidad de toma de decisiones en contexto turbulento. } \\
\text { - Asistencia técnica. } \\
\text { - Intensificación de la competencia. } \\
\text { - Aplicación de nuevas formas de administración. }\end{array}$ \\
\hline Razones de mercado & $\begin{array}{l}\text { - Alianzas en la logística y distribución de los productos. } \\
\text { - Apertura de nuevos mercados. } \\
\text { - Lanzamiento de nuevos productos. } \\
\text { - Elevación de los estándares de calidad. } \\
\text { - Intercambio de información y experiencia entre empresas. } \\
\text { - Aumento de flexibilidad. } \\
\text { - Creación de nuevas empresas. }\end{array}$ \\
\hline
\end{tabular}

Fuente: A partir de (Lozano, 2010)

En consecuencia la Asociatividad es un medio por el cual permite a las empresas su fortalecimiento y crecimiento debido a que se mejora la gestión y así se reducen los riesgos, obteniendo mayores beneficios comunes.

Los diferentes emprendimientos asociativos formados por los artesanos se encuentran con algunas limitaciones durante el proceso de asociarse, entre ellos, se menciona algunos: discusión por obtener el poder, falta de comunicación interna dentro de los equipos de trabajo, desorganización contable, falta de liderazgo en los equipos de trabajo, falta de voluntad para el trabajo en conjunto e incumplimiento de los acuerdos establecidos (Ochoa \& Montoya , 1985)

\subsection{Economía Social y Solidaria}

La Economía Social se empezó a formar con el apoyo de corrientes ideológicas del siglo XIX, iniciada por lucha de los trabajadores, la necesidad de organizarse y como resultado de las carencias y necesidades de una parte de la población. (Bastidas, 2001).

La Asamblea Nacional Constituyente define a la Economía Social y Solidaria (2008) es definida: como un conjunto de formas y practicas económicas, individuales o colectivas, auto gestionadas por sus propietarios, privilegiando al ser humano como sujeto y fin de su actividad, orientada al buen vivir, en armonía con la naturaleza, por sobre el lucro y la acumulación de capital. Por otra parte la 
Economía Social es social cuando supera el corporativismo, la defensa cerrada de sus nuevos intereses particulares, y puede poner sus recursos y capacidades al servicio del desarrollo de nuevos emprendimientos, encarando la resolución de las nuevas necesidades de todos de manera cada vez más amplia y compleja. (Coraggio, 2007). La Economía Social y Solidaria busca que a través de sus formas y prácticas económicas, la satisfacción en común de las necesidades de sus integrantes es decir no buscan el lucro privado sino más bien el bienestar de todos y cada uno de sus integrantes. Por su parte es de suma importancia que el estado fomente la creación de empresas ya que las mismas generan empleo contribuyendo notablemente al desarrollo del país. El desarrollo de la Economía Social y Solidaria implica una lucha cultural y política ya que el fin de la práctica de esta economía es trabajar en conjunto basados en acuerdos básicos que incluyan iniciativas de la ampliación de la igualdad, la justicia, la democracia, la inclusión de todos y todas en armonía con la naturaleza. (Vázquez, 2008). Uno de los aspectos que han contribuido notablemente a nuestro país sin duda es la práctica de la economía social y solidaria, debido a que el trabajo en conjunto permite que las actividades se desarrollen de manera más eficiente, esto gracias al apoyo de diversas instituciones quienes apoyan estas iniciativas con el fin de mejorar el manejo de los negocios.
La Asamblea Nacional Constituyente (2008) menciona algunas de las características de la Economía Social y Solidaria las mismas que son las siguientes:

- La búsqueda de la satisfacción en común de las necesidades de sus integrantes, especialmente, las básicas de autoempleo y subsistencia;

- Su compromiso con la comunidad, el desarrollo territorial y la naturaleza;

- La ausencia de fin de lucro en la relación con sus miembros;

- La no discriminación, ni concesión de privilegios a ninguno de sus miembros;

- La autogestión democrática y participativa, el autocontrol y la auto responsabilidad;

- La prevalencia del trabajo sobre el capital; de los intereses colectivos sobre los individuales; $\mathrm{y}$, de las relaciones de reciprocidad y cooperación, sobre el egoísmo y la competencia.

En cuanto a los distintos principios de la Economía Social y Solidaria se consideran como las normas que debe cumplir para funcionar el sistema económico dentro de los procesos de producción, distribución y comercialización. Por su parte Coraggio (2011) propone una serie de principios que deben considerar dentro de la Economía Social y Solidaria. Los clasifican en 5 grupos según los procesos de la economía: los relativos a la producción, los relativos a la distribución, los relativos a la circulación, los relativos al consumo y otros de carácter trasversal.

El Instituto de Economía Popular y Solidaria (IEPS) mediante Decreto Ejecutivo 1668, del 7 de abril del 2009, con la finalidad de fomentar y promocionar a las personas y organizaciones 
que realizan está alternativa; tiene como visión la construcción del Sistema Económico Social y Solidario del Ecuador con el liderazgo de los actores de la Economía Popular y Solidaria, visibilizados e incluidos en políticas públicas transformadoras, desarrollando procesos productivos basados en la solidaridad, cooperación y reciprocidad, que privilegian al trabajo y al ser humano.

\subsection{Rendimiento empresarial}

El rendimiento se lo define como el resultado de la gestión tanto de la producción como financieras para el logro de los objetivos planteados en la empresa. En el rendimiento el aspecto más importante es la colaboración debido a que es el impulsor clave para obtener éxito en la misma.

En términos financieros se reconoce que la gestión de la calidad puede tener efectos positivos sobre el rendimiento de la organización. En esta línea, Deming (1989) indicó que una mejora de la calidad implica una reducción de costes y una mejora de la productividad que permite a la empresa alcanzar una mayor cuota de mercado y mejorar su competitividad.

Como consecuencia, la calidad puede influir sobre el rendimiento por dos vías complementarias Puede tener: efectos internos a través de los procesos, y efectos externos a través del mercado.
Los efectos internos están relacionados con el funcionamiento interno de las empresas (incremento de la productividad de los trabajadores y de las instalaciones, mejora de la eficiencia, reducción de costes y desperdicios a la hora de prestar el servicio). De esta manera, la calidad puede aumentar la competitividad y su rentabilidad a través de la estandarización de procesos, reducción de desperdicios, servicios más eficientes y disminución de errores. Por su parte, los efectos externos están relacionados con el efecto de la calidad sobre la satisfacción del cliente y la demanda de los servicios (incremento de las ventas y la cuota de mercado, fidelización de los clientes, captación de nuevos clientes, aumento de la satisfacción del cliente, mejora de la imagen de la empresa) (Garvin, 1984).

La importancia del rendimiento o también conocido como el desempeño empresarial es un aspecto muy discutido. Afirma Cortés (2006) que el desempeño o rendimiento puede definirse desde tres enfoques: financiero, financiero y operativo, y efectividad organizacional.

Sugiere Geroski (1992) que la innovación en investigación y desarrollo provoca un aumento de la rentabilidad y el crecimiento de la empresa. Si la innovación implica una mejora del producto y una reducción de costes, la empresa aumentara su beneficio y su cuota de mercado. La innovación tiende a incrementar el 
crecimiento y la eficiencia, pero no implica un mayor beneficio de manera inmediata debido a los costes en que se incurren.

En relación a la innovación se encontraron que las empresas que innovan en sus productos obtenidos poseen una mayor eficiencia, mejora de capacidades para satisfacer a sus clientes, adaptarse a sus necesidades, elevaron su rentabilidad y adaptabilidad. Concluyendo que el tipo de innovación influye en un número mayor si se lo realiza en el producto elaborado. (Gálvez, 2012)

Las diferentes medidas de rendimiento empresarial utilizadas, Santos (1999) las clasifica en tres grupos: medidas financieras o de rentabilidad (margen sobre ventas beneficio, roi), medida operativas (éxito de los nuevos productos, crecimiento de la cuota de mercado), medidas relacionadas con la consecuencia de los objetivos a corto y largo plazo de la empresa.

Lacy (2008) determina que la obtención del alto rendimiento se realiza a través: 1.Posicionamiento y enfoque de mercado: Las empresas de alto rendimiento disfrutan de una destacada clarividencia a la hora de establecer su dirección estratégica, lo cual permite la maximización de los resultados de negocio y situarse en el lugar adecuado en el momento oportuno. 2. Capacidades distintivas: capacidades difíciles de replicar que definen cómo compiten los negocios. 3. Anatomía del rendimiento: elementos esenciales y comunes relacionados con la cultura, el liderazgo y la fuerza laboral de la empresa.

Finalmente para un rendimiento empresarial se considera dos aspectos importantes: el primero es la competencia este factor es crítico debido a que las empresas deben considerar las oportunidad de mejorar tomando en cuenta la innovación, que logra que las empresas busquen alternativas para mejorar su producto y por otro lado el trabajo en equipo es muy fundamental para el funcionamiento de empresa.

\section{Metodologia}

Esta investigación es de tipo descriptiva con un estudio por encuestas, las cuales tuvieron el propósito de obtener información y de esta manera poder enmarcar posibles escenarios de acción a futuro que permitan al Instituto de Economía Popular y Solidaria intervenir y fortalecer a los grupos asociados.

Para la presente investigación se consideró como unidad de investigación a los integrantes del Programa Hilando el Desarrollo que han participado en los últimos 3 años en la provincia de Tungurahua. El Instituto de Economía Popular y Solidaria facilitó un marco muestral de 136 actores o talleres individuales, que en algunos casos se encontraban asociados, en otros casos en proceso de asociación y por ultimo talleres que no han decidido asociarse. Al final se 
tuvo un índice de respuesta del 86\% que equivale a 117 encuestas válidas.

\section{Resultados}

Los resultados de la investigación se muestran

a continuación tomando en cuenta la información más representativa otorgada por las asociaciones del programa gubernamental:

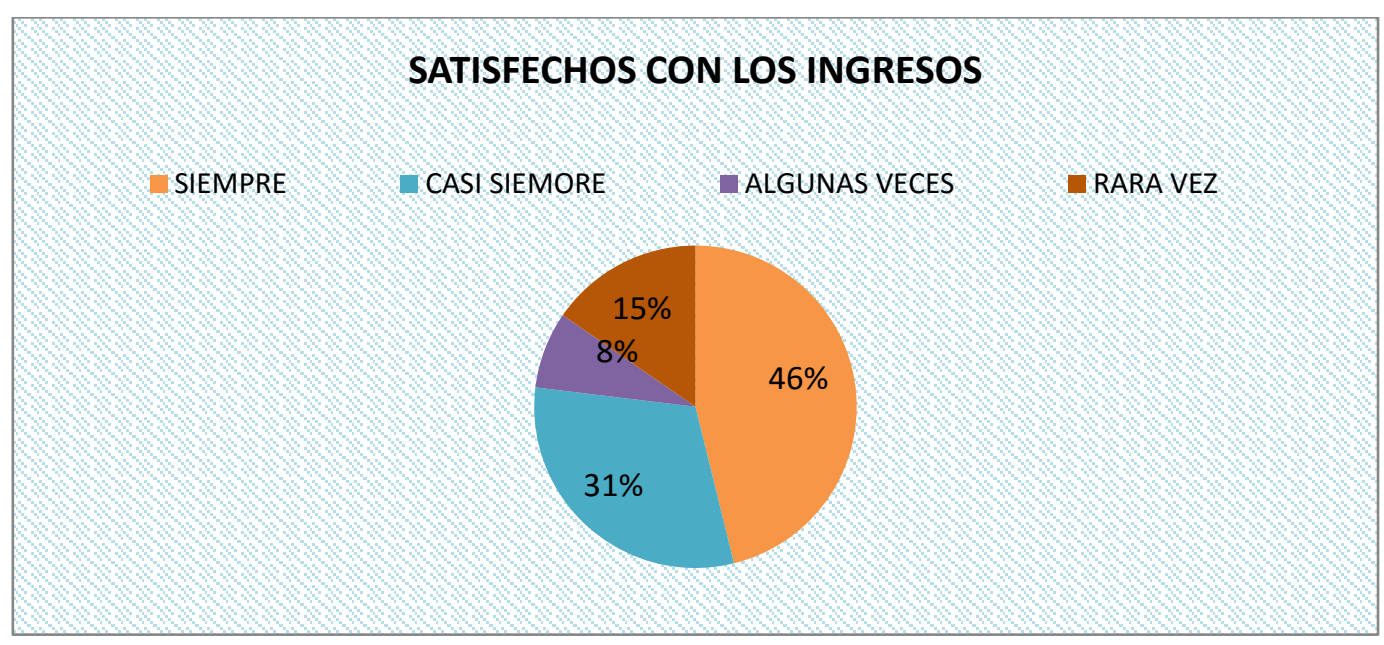

Fuente: Encuestas a integrantes del Programa Hilando el Desarrollo

Evidenciando que la mayoría de los integrantes se encuentren siempre satisfechos con los ingresos percibidos. Los ingresos se obtienen debido a dos aspectos, la calidad y la innovación. En esta línea, Deming (1989) indicó que una mejora de la calidad implica una reducción de costes y una mejora de la productividad que permite a la empresa alcanzar una mayor cuota de mercado y mejorar su competitividad.

Tabla 1. Asociatividad y empleo

Considera que la asociatividad mejorará las fuentes de empleo (familiares, personas cercanas, personas recomendadas o personas con necesidades especiales)

\begin{tabular}{lll} 
ASOCIADO & & \\
SIEMPRE & 16 & $62 \%$ \\
CASI SIEMPRE & 7 & $27 \%$ \\
\hline ALGUNAS VECES & 2 & $8 \%$ \\
RARA VEZ & 1 & $4 \%$ \\
\hline
\end{tabular}

Fuente: Encuestas a integrantes del Programa Hilando el Desarrollo 
Mostrando un alto porcentaje de fuentes de empleo concluimos que cada una de las empresas busca satisfacer necesidades tanto de los clientes como de los empleados. El Estado debe estimular la creación de este tipo de asociaciones. Por su parte es de suma importancia que el Estado fomente la creación de empresas ya que las mismas generan empleo contribuyendo notablemente al desarrollo del país. (Vázquez, 2008)

Tabla 2. Distribución de beneficios

¿Considera usted justa la distribución de los beneficios obtenidos en la asociación?

\begin{tabular}{lll} 
ASOCIADO & & \\
SIEMPRE & 17 & $65 \%$ \\
CASI SIEMPRE & 4 & $15 \%$ \\
\hline ALGUNAS VECES & 1 & $4 \%$ \\
RARA VEZ & 3 & $12 \%$ \\
NUNCA & 1 & $4 \%$ \\
\hline
\end{tabular}

Fuente: Encuestas a integrantes del Programa Hilando el Desarrollo

Para la distribución de beneficios debe tener presente la equidad ya que todos pertenecen a una misma empresa y a un mismo país. En función del pensamiento de Castellanos (2010) la Asociatividad crea condiciones de igualdad para todos sus participantes, de la misma manera permite la interrelación con su entorno empresarial.

Tabla 3. Margen de utilidad

\begin{tabular}{l}
\hline ¿Considera usted que en el programa Hilando el Desarrollo ha obtenido un margen de utilidad \\
aceptable? \\
ASOCIADO \\
SIEMPRE \\
CASI SIEMPRE \\
ALGUNAS VECES \\
RARA VEZ
\end{tabular}

Fuente: Encuestas a integrantes del Programa Hilando el Desarrollo

La Economía Social y Solidaria no busca el lucro privado sino más bien el bienestar de todos y cada uno de sus integrantes. El porcentaje de las utilidades es realmente aceptable el cual contribuye a su fortalecimiento. 
Tabla 4. Productividad

¿Cómo calificaría la productividad alcanzada con la asociación?

ASOCIADO

REGULAR

BUENO

MUY BUENO

$2 \quad 8 \%$

EXCELENTE

$13 \quad 50 \%$

$9 \quad 35 \%$

Fuente: Encuestas a integrantes del Programa Hilando el Desarrollo

Si las empresas asociadas cumplen con lo establecido la productividad será muy eficiente y los ingresos muy altos. Haciendo referencia con la explotación está relacionada con la eficiencia, la selección y la ejecución. Su esencia es la mejora de competencias y tecnologías existentes. La explotación de las competencias es una clave principal para las empresas o rutinas que se están ejecutando (Real, 2006).

Tabla 5. Financiamiento

\begin{tabular}{lll}
\hline Acceso a financiamiento & & \\
\hline EXTREMADAMENTE IMPORTANTE & 5 & $19 \%$ \\
MUY IMPORTANTE & 15 & $58 \%$ \\
IMPORTANTE & 5 & $19 \%$ \\
POCO IMPORTANTE & 1 & $4 \%$ \\
\hline
\end{tabular}

Fuente: Encuestas a integrantes del Programa Hilando el Desarrollo

Todas las empresas necesitan financiarse ya sea para el inicio de una nueva empresa o para la adquisición de nueva maquinaria, esto se lo realiza en instituciones financieras considerando una deuda a corto o a largo plazo. Con ayuda de esta línea Vázquez (2008) la Economía Social y Solidaria busca el bienestar de todos y cada uno de sus integrantes

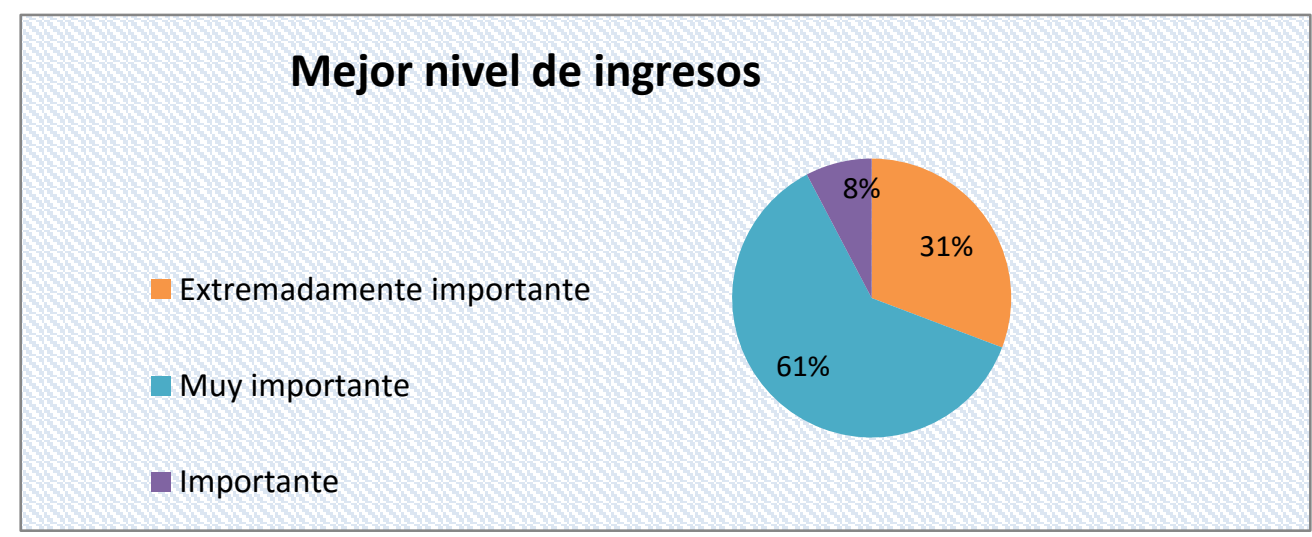

Fuente: Encuestas a integrantes del Programa Hilando el Desarrollo

152 ORTIZ, H.D.; ALVAREZ, E.M. El Rendimiento Empresarial De Las Asociaciones En Un Marco De La Economía Popular Y Solidaria: Estudio En Un Programa Gubernamental. 
En esta línea Geroski (1992) sugieren que la innovación en investigación y desarrollo provoca un aumento de la rentabilidad y el crecimiento de la empresa. Si la innovación implica una mejora del producto y una reducción de costes, la empresa aumentará su beneficio y su cuota de mercado. Dentro de las ventajas al asociarse la mayoría considera que el mejorar el nivel de ingresos es muy importante.

\section{Discusión}

Las ventajas de la Asociatividad son evidentes. Sin embargo, el paso más complicado y decisivo en la construcción de la misma se muestra en el momento no solo de compartir costos, sino también información $\mathrm{y}$ conocimiento (Bedregal, 2014). Siendo fundamental el correcto manejo de la información para llegar al éxito. Obteniendo como resultado un mejor rendimiento debido a dos aspectos, la calidad y la innovación. En esta línea, Deming (1989) indicó que una mejora de la calidad implica una reducción de costes y una mejora de la productividad que permite a la empresa alcanzar una mayor cuota de mercado y mejorar su competitividad.

Para Castellanos (2010) la Asociatividad crea condiciones de igualdad para todos sus participantes, de la misma manera permite la interrelación con su entorno empresarial. Para la distribución de beneficios debe tener presente la equidad ya que todos pertenecen a una misma empresa y a un mismo país. A través de lo establecido se demostró un alto porcentaje de fuentes de empleo concluyendo que cada una de las empresas busca satisfacer necesidades tanto de los clientes como de los empleados. Por su parte es de suma importancia que el Estado fomente la creación de empresas ya que las mismas generan empleo contribuyendo notablemente al desarrollo del país. (Vázquez, 2008).

La Economía Social y Solidaria es uno de las instituciones del Estado que no busca el lucro privado sino más bien el bienestar de todos y cada uno de sus integrantes. El porcentaje de las utilidades es realmente aceptable, de igual forma la distribución de los beneficios es justa, esto contribuye a su fortalecimiento. Además Bedregal (2014) indica que las empresas asociadas comparten costos, capacidades y conocimientos facilitan la productividad que permitirá más eficiencia e ingresos más altos. Real (2006) menciona que: los resultados en la producción serían positivos debido a que el trabajo es en conjunto, siendo la asociatividad una forma de realizar acuerdos de mutuo beneficio.

La Economía Social y Solidaria busca que a través de sus formas y prácticas económicas, la satisfacción en común de las necesidades de sus integrantes es decir no buscan el lucro privado sino más bien el bienestar de todos y cada uno de sus integrantes (Vázquez, 2008). Y esto sucede en el Programa Hilando Desarrollo. 
Uno de los rubros más importantes en las empresas es la necesidad de financiarse ya sea para el inicio de una nueva empresa o para la adquisición de nueva maquinaria, esto se lo realiza en instituciones financieras considerando una deuda a corto o a largo plazo. Siendo establecido por Vásquez (2008) que la Economía Social y Solidaria busca el bienestar de todos sus integrantes en el cumplimiento de sus derechos y objetivos. Finalmente Geroski (1992) sugiere que la innovación en investigación y desarrollo provoca un aumento de la rentabilidad y el crecimiento de la empresa. Si la innovación implica una mejora del producto y una reducción de costes, la empresa aumentará su beneficio y su cuota de mercado.

\section{Conclusiones}

El rendimiento empresarial de las asociaciones se determina por diversos factores en donde uno de los fundamentos es la innovación continua que permite el mejoramiento de sus intereses conjuntos, a través de un intercambio de conocimientos, así como las capacidades potenciales que beneficiarán al desarrollo e impulsarán el incremento de su cuota de mercado en un marco de la economía popular y solidaria que integran una nueva forma de organización dinámica y equilibrada entre la sociedad, estado y mercado (Asamblea Nacional Constituyente, 2008).
Además se determina que la rentabilidad presenta efectos positivos al relacionarlo con la orientación al mercado, en donde influyen muchos aspectos, entre los cuales el análisis de necesidades colectivas y satisfacción de las mismas es un planteamiento común por los miembros que intentan compactar sus intereses de manera más eficiente y viable, dirigiéndose al cumplimiento de sus metas hacia la obtención de resultados concretos.

Por lo que el enfoque financiero ha sido el más empleado y también es el más limitado para medir el rendimiento de una empresa, pues el cumplimiento de todos los objetivos no se puede medir únicamente a través de los resultados financieros que ésta obtiene. Se suelen emplear indicadores como la rentabilidad económica y financiera, la productividad, la solvencia financiera, la eficiencia en costes y el crecimiento en el volumen de ventas (Cortés, 2006).

También se suma a todo ello la asociatividad como una estrategia colectiva que permita promover un cambio en la matriz productiva del país, con el propósito de romper el paradigma empresarial capitalista (individualista) y construir un sistema dinámico en el sector empresarial que cree las condiciones necesarias que conlleven hacia el desarrollo endógeno definido como la capacidad de liderar y conducir su propio 
desarrollo, del sistema productivo actual (Bedoya, 2005)

En consecuencia la generación de mejores rendimientos empresariales se encuentran propuestos según la Asamblea Nacional Constituyente (2008) en la economía popular y

\section{Bibliografía}

Alonzo, M. (2008). sostenibilidad, clave del alto rendimiento en los negocios. Boletin de estudios económicos, 1-21.

Álvarez, M. S. (2002). La orientación al mercado como funete de ventajas competitiivas: antecedentes asociados al equipo directivo y a la estructura organizativa en las empresas industriales. Asturiana de Economía, 215244.

Asamblea Nacional Constituyente. (20 de Octubre de 2008). Asamblea Nacional. Obtenido de Asamblea Nacional: http://www.asambleanacional.gob.ec/si tes/default/files/documents/old/const itucion_de_bolsillo.pdf

Bastidas, O. (2001). La Economia Social y Solidaria: Un intento de definicion. CAYAPA.

Bearden, A. K. (2005). A Meta-Analytic Review and Assessment of it Antecedents and Impact on Perfonrmance. Market Orientationi, 24-41. solidaria que está orientada al buen vivir en armonía con la naturaleza, por sobre el lucro y la acumulación de capital. Siendo el Estado quien impulse la creación de fuentes de empleo a través de la misma que contribuyen a un país más productivo en todas sus áreas.

Bearden., H. K. (2005). Market orientation: a meta- analytic review and assessment of this antecedents and impact on performance. Marketing, 24-41.

Bedoya, F. (2005). Micro y Pequeña industria: Una alternativa para la promoción de la cooperación y la asociatividad. Tendencias, 109-120.

Bedregal, A. E. (2014). La asociatividad como factor de sostenibilidad en asociaciones de productores agricolas y agroindustriales. Revista 27 de la Universidad del Valle, 93-98.

CAF, C. (2007). Electronica colombiana del clase mundial. Fortalecimiento de cluster de electricos $y$ electronicos. Obtenido de PAC: www.caf.com/publicaciones

Calvo, A. \&. (2003). Modelo rbv y rendimiento empresarial:Análisis dicriminante en PYMES Gallegas . Boletines de estudios económico, 503-521.

Castellanos, J. (2010). La asociatividad como fenómeno evolutivo. EAN, 68. 
Chakravarty. (1986). Measuring strategic performance. Strategic Management Journal, 437 a 458.

Coba, E., \& Diaz , J. (2014). El credito de desarrollo humano asociativo en la economia social y solidaria de la provincia de Tungurahua. Revista de Analisis Estadistico, 33-47.

Coraggio. (22 de Agosto de 2007). La Economia Social y la Busqueda de un programa Socialista para el siglo XXI. Obtenido de Facsoandes:

http://www.flacsoandes.edu.ec/web/i magesFTP/1279562108.la_economia_s ocial_y_la_busqueda_de_un_programa _coraggio.pdf

Coraggio. (Julio de 2011). Economia Social y Solidaria. Obtenido de El Trabajo Antes que el Capital: http://www.coraggioeconomia.org/jlc/ archivos $\% 20$ para $\% 20$ descargar/econo miasocial.pdf

Cortés, E. \&. (2006). “A journey toward total quality management through ISO 9000 certification. International Journal of Quality \& Reliability Management, 489 a 508.

Cortés, E. \&. (2006). La medición de los resultados empresariales desde una óptica estratégia. 201 a 264.
Cortés, E. \&. (2006). La relación entre la gestión de la calidad y el rendimiento empresarial en el sector hotelero. papers de turisme, 31 a 47.

Cross, C., \& Berger, M. (2010). La Produccion del trabajo Asociativo. Obtenido de http://base.socioeco.org/docs/la_prod ucasociativa_.pdf

Davila, O. (1993). El dilema de la constitución de actores sociales. Viña de Mar: CIDPA.

Deming, W. (1989). Calidad, Productividad y competitividad. Madrid: Díaz de Santos .

Gálvez, E. \&. (2012). Impacto de la innovacion sobre el rendimiento de la MIPYME. $10-25$.

Garvin, D. (1984). 'What does 'product quality' really. Sloan Management Review, 25-43.

Geroski, P. \&. (1992). Do innovating firms outperform non-innovator? Business Strategy Review, 79-90.

Hall, R. (1992-1993). “'The strat egic analysis of intangi ble resources". Strategic management journal, 135.144.

Holland, J. (1975). Adaptación en un sistema natural y artificial .

Houston, F. (1996). The marketing concept: what it is and what is not. Journal of Marketing, 81-87. 
Hult, R. H. (1998). Innovation, Market Orienation and Organizational Learning: An Integration and Empirical Examination. Marketing, 42-54.

Keith, R. (1960). The Revolution. Marketing, 3538.

Lacy, J. A. (2008). The sustainability imperative. Achieving high perfomance.

Lozano. (2010). Modelos de Asociatividad: estretegias efectivas para el desarrollo de las Pymes. EAN, 175-178.

Maldovan , J., \& Dzembrowski, N. (2009). Asociatividad para el trabajo. Dianet.

Monroy, L., \& David, F. (2010). La Asociatividad como Modelo de Gestion para promover las exportaciones en las Pequeñas y Medianas Empresas. Revista de Relaciones Internacionales Estrategia y Seguridad, 161-191.

Nunes., T. B. (2007). Recorriendo la distancia: cómo las mejores empresas del mundo alcanzan el alto rendimiento.

Ochoa , D., \& Montoya , A. (1985). Administracion de Empesas Asociativs de Productividad Agropecuaria. IICA.

Porter, M. (1991). Towards a Dynamic Theory of Strategy. Strategic management journal, 96-117.
Real, J. (2006). Antecedentes del aprendiaje organizativo como determinante del rendimiento empresarial: El papel del tamaño organizativo como variable moderada. 21

Ripollés, A. B. (2002). Relación entre la orientación al mercado y su escala de medición . Española de investigaciónde Marketing , 165-180.

Rodríguez., J. (2005). Efecto moderador de la tipología estratégica en la relacion orientación mercado. resultados. Europea de direccion y economia de la empresa, 121-138.

Rosales, R. (1997). La asocciatividad como estrategia de fortalecimiento de las PYMES. Caracas: Edición $\mathrm{N}^{\circ} 51$.

Rumelt, R. (1991). How much does industry matter . Academy of management review, 167-185.

Santos, L. Á. (1999). Resultados de la orientacion al mercado: evidencias empiricas de las organizaciones no lucrativas. Investigaciones europeas de direccion y economia de la empresa, 19-40.

Steward, T. (1998). “America's most admired corporations". Fortune, 52.87.

Vázquez, G. (2008). La Economia Social y Solidaria en America Latina. EMES. 
Vegas, J. (2008). Proyecto de cooperacion. Obtenido de

http://www.mincetur.gob.pe/comercio /ueperu/consultora/docs_taller/Presen taciones_Tumbes_y_Piura/1.2.1.2.F1\% 20Asociatividad $\% 2020080912 . p d f$
Weigelt, K. C. (1988). Reputation and corporate Stratery: a Review of Recent Theory and Applications. Strategic Management Journal, 443-454.

158 ORTIZ, H.D.; ALVAREZ, E.M. El Rendimiento Empresarial De Las Asociaciones En Un Marco De La Economía Popular Y Solidaria: Estudio En Un Programa Gubernamental. 